\title{
Tratamiento de radioterapia estereotáxica extracraneal (SBRT) para lesiones múltiples bilaterales de pulmón: isocentro único o isocentros múltiples
}

\section{Stereotactic ablative radiotherapy treatment in multiple bilateral lung lesions: single-isocenter vs multiple-isocenter VMAT approach}

\author{
Caroline Descamps, Egle Aon, Diego Franco, Gustavo Ferraris, Diego Fernandez, Edgar Falco, \\ Edgardo Garrigo
}

Centro Médico Deán Funes, Córdoba, Argentina.

Fecha de Recepción: 18/06/2019 - Fecha de Aceptación: 28/11/2019

La radioterapia estereotáxica extracraneal (Stereotactic Body Radiation Therapy, SBRT) asociada a la arcoterapia volumétrica de intensidad modulada se utiliza desde 2008 para tratar lesiones pulmonares pequeñas.

En este trabajo, se compararon técnicas de planificación para el tratamiento simultáneo de lesiones pulmonares bilaterales utilizando uno o dos isocentros.

Se utilizaron para la técnica con isocentro único dos arcos coplanares y para la técnica multi-isocéntrica, cuatro semiarcos coplanares para cada PTV (planificador Eclipse ${ }^{\mathrm{TM}}$ ). Los planes fueron normalizados para tener 95\% del PTV cubierto por $100 \%$ de la dosis prescrita y se aplicaron las restricciones del TG101 a los órganos de riesgo (OARs). Para comparar las técnicas, se evaluaron índice de conformidad, IC para el 50\% de la dosis prescrita, índice de homogeneidad, dosis máxima a $2 \mathrm{~cm}$ del PTV y las dosis recibidas por los OARs (pulmones, médula, corazón, esófago, tráquea, piel y costillas).

No hubo diferencia significativa en los índices dosimétricos obtenidos y las dosis en los OARs se vieron levemente mejoradas utilizando 2 isocentros pero todos los planes respetaron los criterios de aceptación.

Las dos técnicas aparecen dosimétricamente aceptables y el uso de un único isocentro podría proponerse para lograr planes de calidad con tiempos de tratamiento más cortos en casos de lesiones pulmonares bilaterales.

Palabras clave: SBRT, VMAT, lesiones pulmonares bilaterales, isocentro único/dos isocentros.

Stereotactic Body Radiation Therapy (SBRT) with volumetric-modulated arc-therapy is used since 2008 to treat limited lung disease.

In this study, we compared single-isocenter vs multiple-isocenter approach in case of synchronous bilateral lung lesions treatment.

Patients with bilateral lung disease were calculated in Eclipse ${ }^{\mathrm{TM}}$. For the first approach with one isocenter, two coplanar arcs were used. For the multiple-isocenter approach, four coplanar semi-arcs were used for each isocenter. All plans were normalized to at least $95 \%$ of the PTV receiving $100 \%$ of the prescribed dose and TG101's constraints for the organs at risk (OAR) were adopted. Conformity indices, global maximum doses, maximum doses to any point $2 \mathrm{~cm}$ or greater away from the PTV in any direction and doses at OARs (lung, spinal cord, heart, esophagus, trachea, ribs and skin) were recorded.

No significant differences were observed in dosimetry indices and the OARs absolute doses were slightly better using two isocenters but OARs acceptance criteria was reached for all plans.

The results show that both approaches are dosimetrically allowable. The use of single-isocenter technique can be recommended to obtain excellent plan quality with faster treatment time (single set-up) in bilateral lung lesions SABR.

Key words: SBRT, VMAT, bilateral lung lesions, single-isocenter/multiple-isocenter.

\footnotetext{
*Correspondencia: cdescamps23@yahoo.fr https://doi.org/10.37004/sefm/2020.21.1.001
} 


\section{Introducción}

Las metástasis pulmonares definen la enfermedad oncológica en estadío avanzado, como resultado de diseminación hematógena del sitio primario tumoral. Representan un mal pronóstico para el paciente y una esperanza media de vida limitada. El tratamiento habitual es administrar quimioterapia sistémica y/o terapia molecular dirigida a pacientes que presentan metástasis a distancia, considerando a la mayoría de ellos portadores de una enfermedad incurable a pesar de la terapia instaurada.

Algunos pacientes tienen metástasis a distancia en pocos sitios. En 1995, Hellman y Weichselbaum ${ }^{1,2}$ propusieron un estado intermedio de metástasis denominado "oligometástasis", en el que el número y los sitios de los tumores metastásicos son limitados, generalmente hasta 5 lesiones. Aunque la extirpación quirúrgica se considera un tratamiento radical para pacientes con oligometástasis pulmonares, muchos son inoperables. La radioterapia estereotáxica extracraneal (SBRT) aparece entonces como un tratamiento alternativo para la enfermedad oligometastásica ${ }^{3-5}$ y se utiliza ampliamente en todo el mundo.

Niibe y su equipo definen luego el concepto de oligo-recurrencia 6 (pacientes con menos de 5 lesiones y con enfermedad controlada en el sitio primario del tumor) y recientemente, presentaron sus resultados con SBRT en pacientes con oligo-recurrencia, reportando índices favorables de sobrevida a largo plazo en pacientes con oligo-recurrencia pulmonar. ${ }^{7}$

La SBRT de pulmón, en caso de múltiples lesiones, presenta nuevos desafíos relacionados a los movimientos de las lesiones entre sí y con los órganos de riesgo adyacentes durante el ciclo de la respiración.

Uno de los parámetros de planificación es la elección de la cantidad de isocentros utilizados ya que el uso de múltiples isocentros conlleva a tratamientos más largos (set-up diferentes para cada isocentro) y por lo tanto a errores de posicionamiento y posibles movimientos intra-tratamiento del paciente y de sus órganos. Varios equipos mostraron que el uso de un único isocentro para el tratamiento de múltiples metástasis intra-craneal permite reducir considerablemente el tiempo de tratamiento sin degradar la calidad del tratamiento ${ }^{8,9}$ y más recientemente, se estudió la posibilidad de tratar múltiples lesiones de pulmón con un isocentro único. ${ }^{10,11}$

En este estudio, se seleccionaron pacientes con estadío IV, M1 con dos lesiones (una lesión ubicada en cada pulmón, oligo-recurrencia y oligometastásicos) a los cuales se planificó un tratamiento de SBRT en nuestra institución y, de forma retrospectiva, se compararon planes con un isocentro único o con dos isocentros (un isocentro en cada pulmón).

\section{Material y métodos}

\section{Simulación del paciente y delineación de los volúmenes}

En este trabajo, se realizó un estudio retrospectivo de seis pacientes con lesiones pequeñas ubicadas en ambos pulmones. La Tabla 1 resume los datos para cada paciente de la localización de las lesiones, la distancia entre lesiones y el volumen de los PTV totales.

Para una buena inmovilización de los pacientes y una perfecta reproducibilidad de su posicionamiento en cada sesión de tratamiento, se utilizó el sistema para inmovilización y posicionamiento InmoSBRT (Sedetech*, Buenos Aires, Argentina). Los pacientes fueron ubicados en posición supina en una colchoneta de vacío sobre la camilla indexable del sistema y los apoya-piernas y pies se ajustaron según las medidas de cada paciente. Se utilizó un accesorio para sostener los brazos del paciente arriba de su cabeza. Para regular los movimientos del diafragma en inspiración y exhalación, se utilizó un arco para compresión abdomino-torácica, ajustable en altura según el tamaño del paciente. ${ }^{12}$

Con el objetivo de reducir los movimientos relacionados con la respiración y en consecuencia ajustar los márgenes utilizados posteriormente durante la planificación, se realizaron tres tomografías con un tomógrafo multicorte GE Light Speed ULTRA (GE Healthcare, Waukesha, WI, USA). La primera adquisición se realizó en respiración libre suave. Luego sobre la zona adyacente a las lesiones, se realizaron una adquisición en inspiración leve y otra en espiración leve. Se fusionaron las tres adquisiciones en el sistema de planificación (TPS) Eclipse ${ }^{\mathrm{TM}}$ (Varian Medical Systems, Palo Alto, CA, USA). Se dibujaron los volúmenes blancos (volúmenes tumor macroscópicos) en cada una de las adquisiciones y la unión de esos volúmenes (volumen blanco interno). Para tomar en cuenta las incertidumbres del set-up, de la máquina de tratamiento y de los movimientos de las lesiones, se agregó un margen de $5 \mathrm{~mm}$ para obtener los volúmenes blancos de planificación (PTVs). Se dibujaron también los órganos de riesgo (OARs) cercanos a las lesiones: pulmones sanos, médula, tráquea, corazón, esófago, piel y costillas.

\section{Planificación del tratamiento}

Los planes fueron calculados con el sistema de planificación de tratamiento Eclipse v11.0. Se utilizó el algoritmo de cálculo AAA con una rejilla de cálculo con una resolución de $2 \mathrm{~mm}$. Para cada paciente se calcularon dos planes: con un isocentro único o con dos

\footnotetext{
*http://sedetech.com/inmosbrt/
} 
Tabla 1. Datos de las lesiones de los seis pacientes (LII: Lóbulo inferior izquierdo, LID: Lóbulo inferior derecho, LMD: Lóbulo medio derecho, LSD: Lóbulo superior derecho, LSI: Lóbulo superior izquierdo).

\begin{tabular}{|c|c|c|c|c|}
\hline \multirow{2}{*}{ Paciente } & \multicolumn{2}{|c|}{ Localización } & \multirow{2}{*}{$\begin{array}{c}\text { Distancia entre PTVs } \\
(\mathrm{cm})\end{array}$} & \multirow{2}{*}{$\begin{array}{l}\text { Volumen PTV Total } \\
\left(\mathrm{cm}^{3}\right)\end{array}$} \\
\hline & PTV1 & PTV2 & & \\
\hline 1 & LII. Central & LID Periférico & 8.1 & 42.6 \\
\hline 2 & LII Periférico & LID Periférico & 9.7 & 38.9 \\
\hline 3 & LMD Central & Hilio Pulm. Izq. & 11.6 & 48.1 \\
\hline 4 & Hilio Pulm. Izq. & LID Periférico & 19.0 & 42.7 \\
\hline 5 & LSD Periférico & LSI Periférico & 11.2 & 39.1 \\
\hline 6 & LSD Periférico & LSI Periférico & 11.8 & 40.0 \\
\hline
\end{tabular}

isocentros para un acelerador Trilogy (Varian Medical Systems, Palo Alto, CA, USA) asociado a un colimador multiláminas (MLC) Millennium 120 (80 láminas interiores y 40 exteriores que proyectan en el plano isocéntrico un ancho de 0.5 y $1.0 \mathrm{~cm}$, respectivamente). La dosis prescripta fue de 50 Gy en 5 fracciones de 10 Gy.

Para el primer plan, se utilizó un isocentro único ubicado entre las dos lesiones (centro de masa de la suma de los dos volúmenes). Se generó un plan de arcoterapia volumétrica de intensidad modulada (VMAT) con un haz de 6 MV con dos arcos coplanares de 360 grados cada uno. La rotación del colimador fue de 45 y 315 grados respectivamente.

Para el segundo plan, dos isocentros ubicados en el centro geométrico de cada una de las lesiones fueron utilizados y se generaron para cada lesión cuatro semiarcos de 180 grados cada uno (dos con colimador a 45 grados y dos con colimador a 315 grados).

Todos los planes fueron optimizados permitiendo la variación de la velocidad de la rotación del gantry, de la tasa de dosis y de la posición del MLC. El parámetro de objetivo a los tejidos normales (NTO) se utilizó para controlar los gradientes de dosis de los planes. Para la optimización de los planes con múltiples isocentros, se tomó en cuenta la contribución de cada uno de los isocentros sobre el otro. Los dos planes se normalizaron para que 95\% del volumen blanco reciba $100 \%$ de la dosis prescripta. La Fig. 1 muestra los dos tipos de planificación para un mismo paciente (isocentro único a la izquierda, dos isocentros a la derecha).

\section{Evaluación de los planes}

Cada plan fue evaluado en término de cobertura de los volúmenes blancos con el índice de conformidad (IC) y el índice de homogeneidad $(\mathrm{IH})$ y en término de dosis absorbidas intermedias y bajas con el índice de conformidad definido para el 50\% de la dosis absorbida de prescripción $\left(\mathrm{IC}_{50}\right)$ y la dosis absorbida máxima a $2 \mathrm{~cm}$ del borde del PTV $\left(\mathrm{D}_{2 \mathrm{~cm}}\right)$. Se comparó también la cantidad de UMs totales para tratar las dos lesiones. Finalmente, se registró la dosis recibida por los OARs.

El índice de conformidad de los PTVs fue calculado utilizando la definición del ICRU $62^{13}$ a partir del volumen de la isodosis de prescripción dividido por el volumen del PTV. En un caso ideal, con una cobertura perfecta, el IC debe ser igual a uno. Según el comité de la RTOG (Radiation Therapy and Oncology Group) en lo que concierne a tumores pulmonares en pacientes con

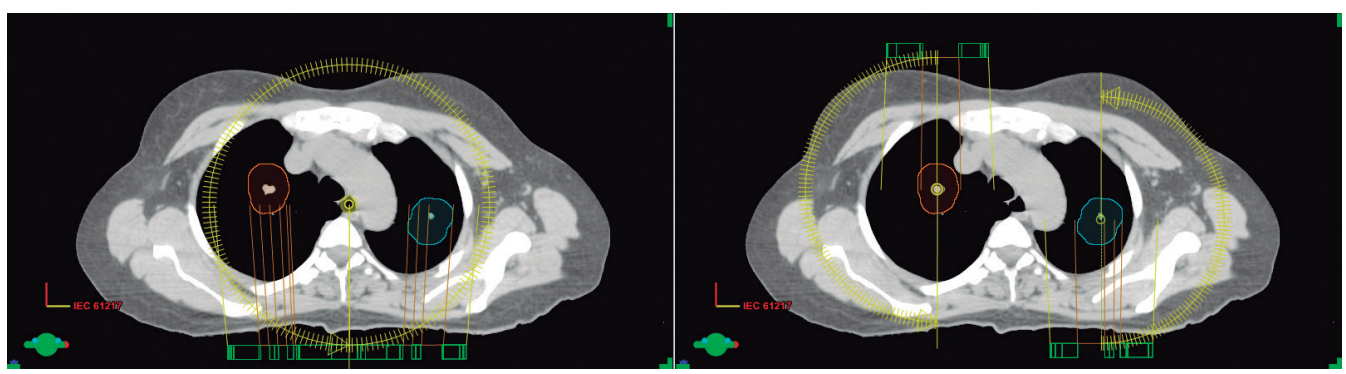

Fig. 1. Izquierda: Planificación con isocentro único ubicado entre las dos lesiones utilizando arcos completos; Derecha: Planificación con 2 isocentros ubicados al centro de cada lesión utilizando semi-arcos de 180 grados. 
tumores únicos (RTOG 0813 y RTOG 0915), el objetivo es tener el IC inferior a 1.2 y el plan será considerado como aceptable si el IC es inferior a 1.5. El índice de homogeneidad se define como la relación entre la dosis absorbida máxima dividida por la dosis absorbida de prescripción. El valor máximo permisible en nuestra institución al utilizar una técnica con modulación de la dosis es del $115 \%$ de la dosis de prescripción con un ideal inferior a $110 \%$.

Para evaluar las dosis absorbidas fuera del PTV y particularmente las dosis intermedias y bajas en relación a la dosis de prescripción, se registró para cada plan el $\mathrm{IC}_{50}$ definido por el volumen de la isodosis del $50 \%$ de la dosis absorbida de prescripción respecto al volumen del PTV. El valor recomendado en los protocolos de la RTOG para pacientes con tumores únicos pequeños va idealmente de 3 a 6 según el volumen del PTV y de 3.7 a 7.5 con una desviación menor. Para asegurar que las dosis recibidas lejos del PTV son suficientemente bajas, se evaluó para cada plan el $D_{2 \mathrm{~cm}}$, definido como la dosis absorbida máxima recibida a $2 \mathrm{~cm}$ del borde del PTV en todas las direcciones. Idealmente, se busca que la isocurva del $50 \%$ quede dentro de los $2 \mathrm{~cm}$ alrededor del PTV.

En lo que concierne a los OARs, se optimizaron los planes siguiendo las restricciones recomendadas por el informe de Benedict et al. en el TG101 de la Asociación Americana de Física Médica (AAPM) ${ }^{14}$ Para permitir la comparación entre los planes se anotaron los volúmenes de pulmón que reciben 5,10 y 20 Gy ( $V_{5 G y}, V_{10 G y}$ y $\left.V_{20 G y}\right)$ y las dosis que reciben 1000 y $1500 \mathrm{cc}\left(\mathrm{D}_{1000 \mathrm{cc}}\right.$ y $\left.D_{1500 c c}\right)$. Los volúmenes de pulmones que reciben 5 y 20 Gy son internacionalmente utilizados ya que son buenos parámetros predictivos de riesgo de neumonitis inducida por radiación. Los valores ideales tienen que ser $V_{5 G y}$ inferior a $40 \%$ y $V_{20 G y}$ inferior a $10 \%$. Según el TG101, las dosis $D_{1000 c c}$ y $D_{1500 c c}$ deben quedar menores a 13,5 Gy y 12,5 Gy respectivamente. También se registraron las dosis que recibe $0.35 \mathrm{cc}$ y $1.2 \mathrm{cc}$ de la médula $\left(D_{0.35 c c}\right.$ y $\left.D_{1.2 c c}\right)$, la dosis máxima y la dosis que recibe $15 \mathrm{cc}$ del corazón $\left(D_{\max }\right.$ y $D_{15 c c}$ ), la dosis máxima y la dosis que recibe $5 \mathrm{cc}$ del esófago $\left(D_{\max } \mathrm{y}\right.$ $D_{5 c c}$ ), la dosis máxima y la dosis que recibe $4 \mathrm{cc}$ de la tráquea $\left(D_{\max }\right.$ y $\left.D_{4 c c}\right)$, la dosis máxima y la dosis que recibe $10 \mathrm{cc}$ de la piel $\left(D_{\max }\right.$ y $\left.D_{10 c c}\right)$ y finalmente, la dosis máxima y la dosis que recibe $1 \mathrm{cc}$ de las costillas $\left(D_{\max }\right.$ y $\left.D_{1 c c}\right)$. Para cada paciente, se calculó la diferencia entre plan calculado con un único isocentro y plan multi-isocéntrico, y para los seis pacientes se determinaron el valor promedio y la desviación estándar de cada parámetro para único isocentro por un lado o dos isocentros por otro lado.

\section{Resultados}

\section{Índices de conformidad}

Todos los PTVs utilizados en este trabajo tuvieron un volumen similar (promedio de $21.0 \mathrm{cc} \pm 3.5 \mathrm{cc}$ con un rango entre 13.8 y $26.4 \mathrm{cc}$ ). Para los planes calculados con un isocentro único, el índice de conformidad promedio fue de $1.01 \pm 0.02$ (rango 0.99-1.03) contra $1.02 \pm 0.02$ (rango 0.99-1.04) para dos isocentros por lo cual la cobertura de los volúmenes blancos en las dos técnicas de planificación resultó ser parecida y muy aceptable. La dosis máxima de cada plan se ubicó para ambos técnicas siempre en el volumen blanco y mostró un leve mejoría en caso de utilizar 2 isocentros (diferencia del IH 1 isocentro contra 2 isocentros de 1.4\%).

En lo que concierne a las dosis bajas e intermedias, las 2 técnicas dan resultados similares. El IC 50 promedio fue de $5.3 \pm 0.3$ para 1 isocentro contra $5.2 \pm 0.6$ para 2 isocentros y el $D_{2 \mathrm{~cm}}$ fue de $51.7 \% \pm 1.8 \%$ para 1 isocentro contra $50.8 \% \pm 1.3 \%$ para 2 isocentros. Para algunos pacientes, el $\mathrm{IC}_{50}$ y el $\mathrm{D}_{2 \mathrm{~cm}}$ fueron mejorados con 2 isocentros y en otros pacientes, con un solo isocentro.

Tabla 2. Parámetros dosimétricos obtenidos para los seis pacientes con uno o dos isocentros (IC, IH, IC $50, D_{2} \mathrm{~cm}$ y cantidad total de UMs).

\begin{tabular}{c|c|c|c|c|c|c|c|c|c|c}
\multirow{2}{*}{ Paciente } & \multicolumn{2}{|c|}{ IC } & \multicolumn{2}{|c|}{ IH } & \multicolumn{2}{|c|}{ D2cm } & \multicolumn{3}{c|}{ IC50\% } & \multicolumn{2}{c}{ UMs Totales } \\
\cline { 2 - 11 } & 1 iso & 2 isos & 1 iso & 2 isos & 1 iso & 2 isos & 1 iso & 2 isos & 1 iso & 2 isos \\
\hline $\mathbf{1}$ & 1.03 & 1.03 & 114.0 & 110.7 & 52.7 & 52.9 & 5.1 & 4.8 & 2882 & 4910 \\
\hline $\mathbf{2}$ & 1.03 & 1.04 & 110.7 & 112.3 & 48.0 & 50.6 & 5.0 & 4.7 & 3316 & 5067 \\
\hline $\mathbf{3}$ & 1.01 & 1.01 & 111.8 & 110.2 & 52.4 & 49.8 & 5.0 & 5.1 & 3073 & 4874 \\
\hline $\mathbf{4}$ & 0.99 & 1.00 & 116.1 & 110.2 & 52.9 & 49.1 & 5.3 & 4.6 & 2832 & 4682 \\
\hline $\mathbf{5}$ & 1.00 & 1.02 & 111.6 & 112.0 & 52.4 & 50.9 & 5.6 & 5.9 & 3043 & 4730 \\
\hline $\mathbf{6}$ & 1.01 & 0.99 & 113.3 & 113.0 & 51.8 & 51.6 & 5.8 & 5.7 & 2955 & 5085
\end{tabular}




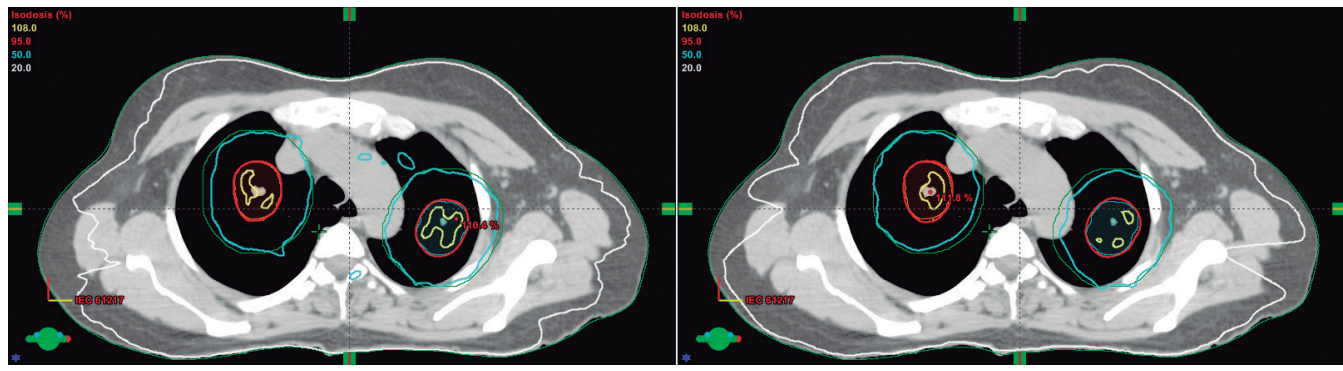

Fig. 2. Planificación con isocentro único (Izquierda) y con dos isocentros (Derecha). Se presentan isocurvas representativas y el contorno de los PTV y del contorno $D_{2 c m}$ (en verde).

El uso de un isocentro único permitió bajar de casi 40\% la cantidad de UMs necesarias para el tratamiento de las dos lesiones lo que conlleva a tratamientos más cortos, adicionado al hecho que solo será necesario un único set-up. Todos los resultados están resumidos en la Tabla 2.

La Fig. 2 muestra, a modo de ejemplo, la dosimetría obtenida para uno de los pacientes. Para los dos abordajes, las isocurvas de referencia ( $95 \%$ en rojo) fueron idénticas; la variación del volumen recibiendo 95\% de la dosis fue de $0.2 \%$. La isocurva del $50 \%$ de la dosis (en celeste en la Fig. 2) apareció levemente menor con el uso de dos isocentros, con una variación promedia de $1.9 \%$ entre los dos planes y la isocurva del $20 \%$ de la dosis prescrita (en blanco) fue $9.5 \%$ menor con dos isocentros que con uno solo. También figura en verde el contorno del $D_{2 \mathrm{~cm}}$, adentro del cual en lo ideal tiene que estar confinada la isocurva celeste del $50 \%$ de la dosis prescrita.

\section{Órganos de riesgo}

Todos los planes cumplieron con los parámetros de aceptación utilizados en nuestra institución. En el caso de los pulmones, los valores de los volúmenes recibiendo 5, 10 y 20 Gy están resumidos en la Tabla 3. Se calcularon los promedios de porcentaje de volumen para un isocentro único por un lado y dos isocentros por el otro, y luego las diferencias entre ambos grupos de planes. Se mostró que el uso de un único isocentro lleva a un ligero aumento de los volúmenes recibiendo
5 Gy, 10 Gy y 20 Gy (las diferencias entre planes calculados con isocentro único y con múltiples isocentros fueron $V_{5 G y}=8.1 \pm 7.0 \%, V_{10 G y}=3.3 \pm 2.9 \% V_{20 G y}=$ $0.7 \pm 0.7 \%$ ). De la misma forma, como se puede ver en la Tabla 3, las dosis $D_{1000 c c}$ y $D_{1500 c c}$ son levemente más bajas con dos isocentros que con uno pero con valores muy por debajo de las recomendadas por el TG101.

En lo que concierne los otros OAR considerados en nuestro trabajo, los resultados están detallados en la Tabla 4. Cabe destacar que, debido al estado avanzado de enfermedad de los pacientes presentados en este trabajo y siguiendo las recomendaciones de la RTOG, se permitió superar el límite de dosis recomendado a las costillas ( $D_{\max }<43$ Gy y $D_{1 c c}<35$ Gy) en uno de los pacientes que presentaba una lesión muy cercana a las costillas al fin de garantizar un buen control de la enfermedad con una mejor cobertura del PTV. Para los seis pacientes, en promedio, la dosis recibida por las costillas fue muy similar en ambos técnicas $\left(D_{\max }=37.9 \pm 14.2 \mathrm{~Gy}\right.$ y $D_{1 \mathrm{cc}}=29.1 \pm 9.5$ Gy con un isocentro único contra $D_{\max }=37.2 \pm 10.2 \mathrm{~Gy}$ y $D_{1 \mathrm{cc}}=28.6 \pm 10.1$ Gy con dos isocentros).

El uso de la técnica VMAT con múltiples "puertas" de entrada del haz de irradiación permite bajar de manera considerable la dosis recibida por la piel que, con ambos técnicas fue por debajo de las recomendaciones internacionales ( $D_{\max }<39.5$ Gy y $D_{10 c c}<36.5$ Gy) quedando las dosis obtenidas inferiores utilizando dos isocentros.

En el caso de la médula y el esófago, el uso de un isocentro único se traduce por el aumento leve de las dosis recibidas ( $D_{\max }, D_{0.35 c c}$ y $D_{1.2 c c}$ para la médula

Tabla 3. Resultados dosimétricos obtenidos para el pulmón.

\begin{tabular}{c|c|c|c|c|c} 
& V5Gy (\%) & V10Gy (\%) & V20Gy (\%) & D1000cc(Gy & D1500cc (Gy) \\
\hline Isocentro único & $33.4 \pm 14.0$ & $19.6 \pm 4.9$ & $6.5 \pm 0.8$ & $5.4 \pm 2.5$ & $3.0 \pm 1.9$ \\
\hline Dos isocentros & $25.3 \pm 8.5$ & $16.3 \pm 4.3$ & $5.7 \pm 0.6$ & $3.4 \pm 1.8$ & $1.5 \pm 1.0$ \\
\hline Dif. (1iso-2isos) & $8.1 \pm 7.0$ & $3.3 \pm 2.9$ & $0.7 \pm 0.7$ & $2.0 \pm 1.0$ & $1.5 \pm 1.0$
\end{tabular}


Tabla 4. Resultados dosimétricos para la médula, el corazón, el esófago, la tráquea, la piel y las costillas. Para cada parámetro analizado, se presentan los valores objetivos utilizados y el promedio y la desviación estándar de la diferencia de dosis obtenidas con un isocentro contra dos isocentros.

\begin{tabular}{c|c|c|c|c}
\multirow{2}{*}{ 0ARs } & Parámetro & $\begin{array}{c}\text { Promedio (Gy), } \\
1 \text { iso }\end{array}$ & $\begin{array}{c}\text { Promedio (Gy), } \\
\text { 2 isos }\end{array}$ & $\begin{array}{c}\text { Dif. (1iso-2isos) } \pm \\
\text { Desv. Est. (Gy) }\end{array}$ \\
\hline \multirow{4}{*}{ Médula } & $D_{\max }<30$ Gy & $21.7 \pm 3.6$ & $17.0 \pm 4.5$ & $4.7 \pm 2.3$ \\
\cline { 2 - 5 } & $D_{0.35 c c}<23$ Gy & $20.1 \pm 3.5$ & $15.4 \pm 4.1$ & $4.7 \pm 1.8$ \\
\cline { 2 - 5 } & $D_{1.2 c c}<14.5$ Gy & $18.2 \pm 3.4$ & $13.9 \pm 3.6$ & $4.3 \pm 1.8$ \\
\hline \multirow{3}{*}{ Corazón } & $D_{\max }<38$ Gy & $30.0 \pm 24.0$ & $29.2 \pm 25.1$ & $0.8 \pm 2.4$ \\
\cline { 2 - 5 } & $D_{15 c c}<32$ Gy & $17.0 \pm 12.0$ & $14.4 \pm 11.7$ & $2.6 \pm 1.9$ \\
\hline \multirow{3}{*}{ Esófago } & $D_{\max }<35$ Gy & $25.5 \pm 4.6$ & $21.6 \pm 7.6$ & $3.9 \pm 3.5$ \\
\cline { 2 - 5 } & $D_{5 c c}<19.5$ Gy & $17.1 \pm 3.8$ & $14.5 \pm 4.8$ & $2.6 \pm 1.9$ \\
\hline \multirow{3}{*}{ Tráquea } & $D_{\max }<40$ Gy & $16.4 \pm 8.5$ & $15.7 \pm 9.2$ & $0.7 \pm 1.4$ \\
\cline { 2 - 5 } & $D_{4 c c}<16.5$ Gy & $9.9 \pm 7.2$ & $9.1 \pm 8.4$ & $0.8 \pm 1.4$ \\
\hline \multirow{3}{*}{ Piel } & $D_{\max }<39.5$ Gy & $20.0 \pm 4.0$ & $16.8 \pm 2.2$ & $3.2 \pm 2.0$ \\
\cline { 2 - 5 } & $D_{10 c c}<36.5$ Gy & $13.7 \pm 2.8$ & $11.2 \pm 1.6$ & $2.6 \pm 1.4$ \\
\hline \multirow{2}{*}{ Costillas } & $D_{\max }<43$ Gy & $37.9 \pm 14.2$ & $37.2 \pm 14.6$ & $0.7 \pm 2.4$ \\
\cline { 2 - 5 } & $D_{1 c c}<35$ Gy & $29.1 \pm 9.5$ & $28.6 \pm 10.1$ & $0.5 \pm 2.8$
\end{tabular}

y $D_{\max }$ y $D_{5 c c}$ para el esófago) pero en ambos casos (isocentro único o dos isocentros), los planes fueron considerados como aceptables para un eventual tratamiento. Para todos los pacientes, las dosis recibidas por el corazón y la tráquea fueron muy similares y en algunos planes fueron más bajas con el uso de un solo isocentro y en otros con el uso de dos isocentros por lo cual se podría pensar que la influencia de la técnica utilizada es relativamente baja y depende en parte de la ubicación entre los OARs y los volúmenes blancos. La alta desviación estándar de las dosis recibidas por el corazón, en particular, fue debida a la ubicación de las lesiones que en dos pacientes fueron muy próximas al corazón y en otros dos muy alejadas.

\section{Discusión}

En este trabajo mostramos nuestra experiencia en tratamiento de pacientes con lesiones en ambos pulmones, con tratamiento ablativo de radioterapia hipofraccionado a razones de 5 fracciones de 10 Gy cada una. El problema en pacientes con múltiples lesiones utilizando múltiples isocentros es que los tratamientos son largos ya que es necesario realizar desplazamientos e imágenes (nuevo set-up) para cada uno de los isocentros. Varios equipos propusieron primero para tratamiento intracraneal y más recientemente para tratamientos extra-craneales la posibilidad de utilizar un único isocentro para tratar las diferentes lesiones sin degradar la calidad del plan y así disminuir los tiempos de tratamiento. En el caso de lesiones en pulmón, algunos grupos trabajaron con pacientes teniendo dos o más lesiones en un solo pulmón y mostraron que el uso de un único isocentro aparece como una buena opción ${ }^{15,16}$ utilizando IMRT con gantry fijo, tomoterapia o técnica VMAT.

En nuestro caso, el hecho de tener lesiones en ambos pulmones agrega un factor al momento de empezar la planificación utilizando una técnica de arcoterapia ya que cuando se utilizan dos isocentros ubicados en el centro de cada volumen y semi arcos, se espera tener una mejor protección de los órganos de riesgo particularmente centrales. En nuestro estudio, comparamos una planificación convencional con dos isocentros y cuatro semi-arcos contra una planificación con un único isocentro ubicado entre las dos lesiones (y por lo tanto entre los dos pulmones) utilizando dos arcos completos de 360 grados.

El uso de un único isocentro para tratar varias lesiones permite, como mencionamos anteriormente, reducir los tiempos de tratamiento (un solo set-up y cantidad de UMs reducidas) y en caso de lesiones en pulmones, mejorar el confort del paciente y en particular, los movi- 
mientos intra-tratamiento. En nuestra institución, para limitar al máximo estos movimientos se utilizó también un sistema de compresión torácica.

Todos los planes calculados mostraron excelentes coberturas de las lesiones a tratar y homogeneidad de la dosis adentro de los PTVs, con muy buenos índices de conformidad y dosis máximas obtenidos, permitiendo un control de la enfermedad. El volumen de tejido recibiendo el 95\% fue el mismo en los dos tipos de tratamiento. En lo que concierne a las dosis bajas e intermedias en comparación con la dosis prescripta, tanto el índice $\mathrm{IC}_{50}$ como el $\mathrm{D}_{2 \mathrm{~cm}}$ fueron levemente mejorados con el uso de 2 isocentros pero en todos los planes obtenidos fueron muy aceptables lo que demuestra la calidad de todos los planes calculados.

La dosis recibida por los pulmones sanos aparece de gran importancia, particularmente cuando los dos pulmones presentan lesiones, para limitar las toxicidades relacionadas a la radiación. Los volúmenes de pulmones sanos recibiendo 5 y 20 Gy son buenos indicadores de posible neumonitis radio-inducida. En este trabajo se vio que el uso de dos isocentros permitió bajar levemente esas dosis pero que los planes calculados con un único isocentro resultaron ser totalmente aceptables.

De la misma forma, para los otros órganos de riesgo, el uso de dos isocentros para la planificación mostró una leve mejoría de las dosis recibidas por los tejidos sanos. Cabe destacar que la localización de las lesiones y su proximidad con los órganos de riesgo influyen altamente sobre los resultados obtenidos. Igualmente, todos los planes evaluados (con uno o dos isocentros) fueron aprobados según los criterios aplicados en nuestra institución para una posible irradiación del paciente.

Para permitir un tratamiento óptimo, parece importante que el acelerador utilizado responda a todos los criterios de calidad para tratamientos de SBRT. Cuando se utiliza la técnica VMAT y en el caso de pequeños volúmenes, el correcto posicionamiento del paciente parece primordial, ya que un mínimo error en el posicionamiento del paciente puede llegar a irradiar con muy altas dosis tejidos sanos y subdosar los volúmenes blancos. Para garantizar la calidad de tratamiento, se realiza diariamente, semanalmente, mensualmente y anualmente todos los controles descriptos en el informe TG142 de la AAPM. ${ }^{17}$

\section{Conclusión}

En conclusión, según nuestra experiencia en tratamientos de lesiones (oligo-recurrencia y oligometastásicas) bilaterales en pulmón con dosis ablativas, el uso de un único isocentro para tratar dos sitios aparece como una buena alternativa. Permite reducir considerablemente los tiempos de tratamiento y en consecuencia mejorar la comodidad del paciente logrando así reducir los posibles movimientos del paciente o de sus órganos internos durante cada fracción, conservando una calidad de planificación conforme a los criterios utilizados en nuestro centro.

\section{Bibliografía}

1. Hellman S, Wechselbaum RR. Oligometastases. Journal of Clinical Oncology. 1995;13(1):8-10.

2. Weichselbaum RR, Hellman S. Oligometastases revisited. Nat Rev Clin Oncol 2011;8:378-82.

3. Lodeweges, J.E., Klinkenberg, T.J., Ubbels, J.F., Groen, H.J.M., Langendijk, J.A., and Widder, J. Long-term outcome of surgery or stereotactic radiotherapy for lung oligometastases. J Thorac Oncol. 2017;12:1442-5.

4. Siva S, MacManus M, Ball D. Stereotactic radiotherapy for pulmonary oligometastases: a systematic review. J Thorac Oncol. 2010 Jul;5(7):1091-9.

5. Navarria P, De Rose F, Ascolese AM. SBRT for lung oligometastases: Who is the perfect candidate? Rep Pract Oncol Radiother. 2014;20(6):446-53. doi:10.1016/j.rpor. 2014.11.005

6. Niibe Y, Hayakawa K. Oligometastases and oligorecurrence: the new era of cancer therapy. Jpn J Clin Oncol 2010;40:107-11.

7. Nibbe $Y$, Jingu K, Onishi H. Long-Term Outcome of Surgery or Stereotactic Radiotherapy for Lung Oligo-recurrence. J Thorac Oncol. 2017 Nov;12(11):e191.

8. Clark GM, Popple RA, Prendergast BM, Spencer SA, Thomas EM, Stewart JG, et al. Plan quality and treatment planning technique for single isocenter cranial radiosurgery with volumetric modulated arc therapy. Pract Radiat Oncol (2012) 2:306-13.

9. Nath SK, Lawson JD, Simpson DR, Vanderspek L, Wang JZ, Alksne JF, et al. Single-isocenter frameless intensity-modulated stereotactic radiosurgery for simultaneous treatment of multiple brain metastases: clinical experience. Int J Radiat Oncol Biol Phys (2010) 78:91-7.

10. Sanford $L$ et al. Evaluation of plan quality and treatment efficiency for single-isocenter/two-lesion lung stereotactic body radiation therapy. J Appl Clin Med Phys. 2019 Jan;20(1):118-27.

11. Quan K, Xu KM, Lalonde R, et al. Treatment plan technique and quality for single isocenter stereotactic ablative radiotherapy of multiple lung lesions with volumetric modulated arc therapy or intensity modulated radiosurgery. Front Oncol. 2015;5:1-9.

12. Brandner ED, Chetty IJ, Giaddui TG, Xiao Y, Huq MS. Motion management strategies and technical issues associated with stereotactic body radiotherapy of thoracic and upper abdominal tumors: A review from NRG oncology. Med Phys. 2017;44(6):2595-612.

13. International Commission on Radiation Units and Measurements (ICRU). Prescribing, recording and reporting photon beam therapy. ICRU Report 62. (Supplement to ICRU Report 50). Bethesda, MD: ICRU Publications; 1999.

14. Benedict SH, Yenice KM, Followill D, et al. Stereotactic body radiation therapy: the report of AAPM Task Group 101. Med Phys. 2010;37:4078-100. 
15. Gulam M, Gopal A, Wen N, et al. Single isocenter lung SBRT for multiple PTV lesions. Int J Radiat Oncol Biol Phys. 2014;90:S910-S911

16. Sanford L, Molloy J, Kumar S, et al. Evaluation of plan quality and treatment efficiency for single-isocenter/two-lesion lung stereotactic body radiation therapy. J Appl Clin Med Phys 2018.

17. Klein E, Hanley J, Bayouth J, et al. Task Group 142 Report: QA of Medical Accelerators. Med. Phys. 2009;36:4197212. 


\section{SIEMENS}

\section{Small cells don't have to lead to big problems.}

Siemens answers help doctors detect diseases earlier, saving costs and extending lives.

When diseases are caught early, they make less of an impact on everyone. That's because a large majority of today's healthcare costs go toward treating the late stages of diseases like cancer and heart disease. With Siemens advanced diagnostic technology, doctors can accurately identify these killers earlier. So patients get the treatment they need sooner. Which helps save lives and cuts costs. Wherever there are tough healthcare questions, we're answering them. 